\title{
Suspended-core fiber Bragg grating sensor for directional- dependent transverse stress monitoring
}

\author{
Charles M. Jewart, ${ }^{1}$ Tong Chen, ${ }^{1}$ Eric Lindner, ${ }^{2}$ Julia Fiebrandt, ${ }^{2}$ Manfred Rothhardt, ${ }^{2}$ \\ Kay Schuster, ${ }^{2}$ Jens Kobelke, ${ }^{2}$ Hartmut Bartelt, ${ }^{2}$ and Kevin P. Chen ${ }^{1, *}$ \\ ${ }^{1}$ Department of Electrical and Computer Engineering, University of Pittsburgh, 3700 O'Hara Street, Pittsburgh, Pennsylvania 15261, USA \\ ${ }^{2}$ Institute of Photonic Technology, Department of Fiber Optics, Albert Einstein Str. 9, 07745 Jena, Germany \\ *Corresponding author: pec9@pitt.edu
}

Received February 24, 2011; revised March 30, 2011; accepted April 9, 2011; posted April 11, 2011 (Doc. ID 143072); published June 15, 2011

\begin{abstract}
This Letter presents simulation and experimental results of orientation-dependent transverse stress fiber sensors using fiber Bragg gratings (FBGs) inscribed in four-hole suspended-core fibers. Resonant peak shifts and splitting of FBGs were studied as functions of the applied transverse load and fiber orientation. Both simulation and experimental results revealed that the response of FBGs in suspended-core fibers is sensitive to both the orientation and magnitude of an applied transverse stress. (C) 2011 Optical Society of America

OCIS codes: $\quad 060.2370,060.3735,060.4005,280.4788$.
\end{abstract}

In the past decade, fiber Bragg grating (FBG) sensors have found increasing applications in structural health monitoring [1-5]. Their compact size, field ruggedness, immunity to electromagnetic noise, and multiplexing capability make FBG sensors prime candidates for harsh environment sensing. Currently, most FBG strain gauges used in structural health monitoring are inscribed in standard telecommunications fiber. As strain gauges, the measurement sensitivity and directionality are inherently limited by the symmetric fiber structure and the mechanical properties of the silica material. FBG sensors inscribed in standard fibers cannot measure the orientation of transverse load due to the cylindrical symmetry of the fiber $[3,4]$. To overcome these limitations, air-hole microstructured optical fibers (MOFs) have been developed to improve the functionality of fiber sensors beyond those fabricated in standard fibers. For example, the addition of an air-hole matrix within the fiber cladding enables the development of FBG sensors that can separate temperature and longitudinal-strain-induced FBG shifts [6].

Ingenuity in the air-hole matrix design for MOFs can also be implemented to improve the FBG sensor's response to directionality and sensitivity in measuring transverse stress. When an air-hole microstructured fiber deforms under an applied transverse load, the induced stress throughout the fiber is affected by the air-hole geometry. It has been shown that it is possible to focus or deflect the stress away from the fiber core by proper design of the air-hole matrix [3]. Previous works using two-hole fibers have shown a 4.8 times improvement in transverse strain measurements compared with standard fibers.

The improvement of directionality in strain measurement can be achieved by using highly asymmetric fiber structures. In this Letter, we present FBG sensors inscribed in a four-hole suspended-core fiber [7-9]. FBGs with this fiber structure are more sensitive to the applied transverse load than standard fibers and possess an orientation dependency to transverse loads. With this unique trait, it is possible to create an embedded fiber strain gauge that can simultaneously measure both magnitudes and directions of axial and transverse strains.

The four-hole suspended-core fiber used in this work is fabricated with stack and draw technology []], as shown in Fig. 1. The diameters of the fiber and the air holes are 123.2 and $25 \mu \mathrm{m}$, respectively. The all-silica fiber core is connected to the outer shell with four bridges with a thickness of $0.9 \mu \mathrm{m}$. This highly nonradial symmetric structure ensures good directionality of transverse strain measurement of the FBG inscribed in the fiber core.

The stress distribution throughout the fiber was simulated using a finite element analysis (FEA) model and can then be related to induced birefringence in silica glass using the model developed by Gafsi and El-Sherif [10]. Birefringence data was calculated from the stress distribution using a two-dimensional (2D) plane-strain FEA model (ANSYS v. 11.0). The 2D model was chosen due to the large aspect ratio of the fiber structure. It allows fine mashing to accurately calculate the stress distribution in air bridges and the fiber core in a reasonable time. Simulations of the induced birefringence under a transverse load of $50 \mathrm{~N} / \mathrm{cm}$ for the two fiber orientations (bridge and hole under the vertical applied load) are shown in Figs. 2(a) and 2(b). The birefringence $(B=$ $\Delta n_{x}-\Delta n_{y}$ ) induced in the vertical bridge orientation is $5.948 \times 10^{-4}$, which is more than 22 times larger than that for the vertical hole orientation of $2.624 \times 10^{-5}$.

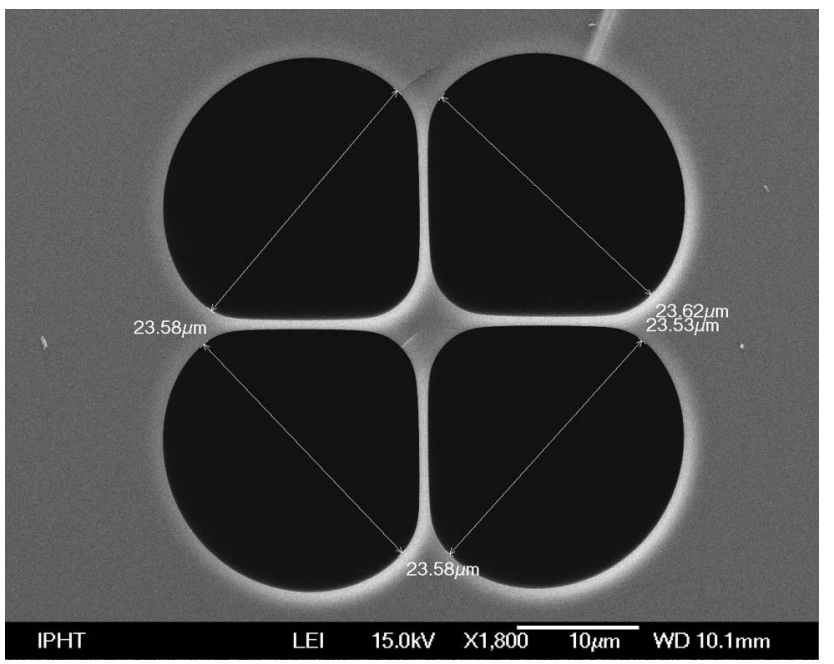

Fig. 1. Scanning electron microscope photograph of four-hole suspended-core fiber. 
To verify the simulated results, a directional compression test was performed on the four-hole suspended-core fiber with FBGs included. Because of the pure silica material of the fiber, conventional UV writing of Bragg gratings is not possible and also hydrogen loading is difficult because of the fast out diffusion of the small core region. Therefore, all gratings were generated by using a femtosecond laser inscription technique. A frequency tripled Ti:sapphire laser working at $266 \mathrm{~nm}$ was used together with a Talbot interferometer configuration, described in [11]. The fabricated gratings had a spectral width between 0.4 and $0.6 \mathrm{~nm}$ and a reflectivity between $50 \%$ and $70 \%$.

The configuration of the loading test experiment was similar to that used in [5] and is briefly described here. First, the suspended-core fiber was mounted on a rotational stage to adjust the orientation of the fiber to the external load. The orientation of the air holes was via a CCD microscope mounted at the end of the fiber. The test fiber and a dummy fiber were mounted between two flat, polished metal plates. The transverse stress was applied via a spring-loaded apparatus and monitored via

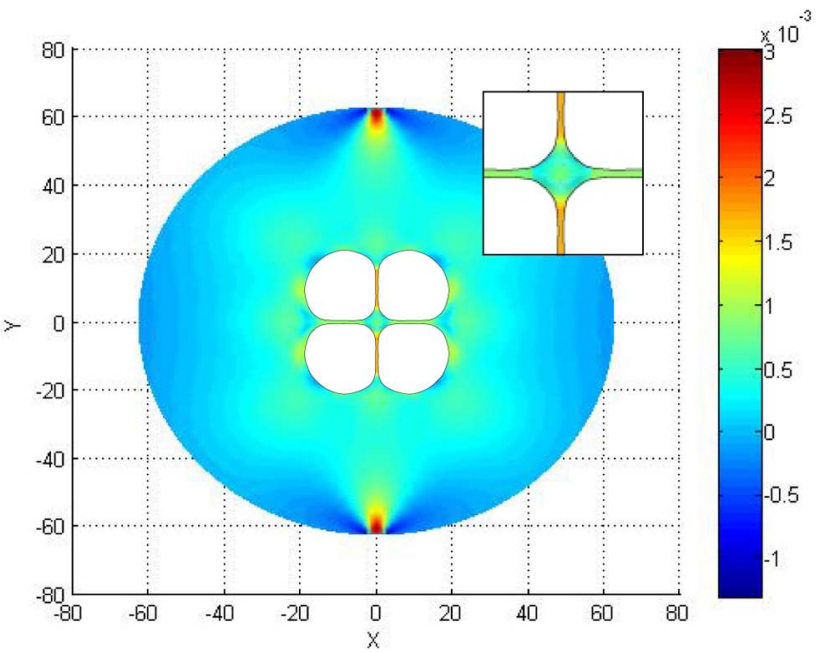

(a)

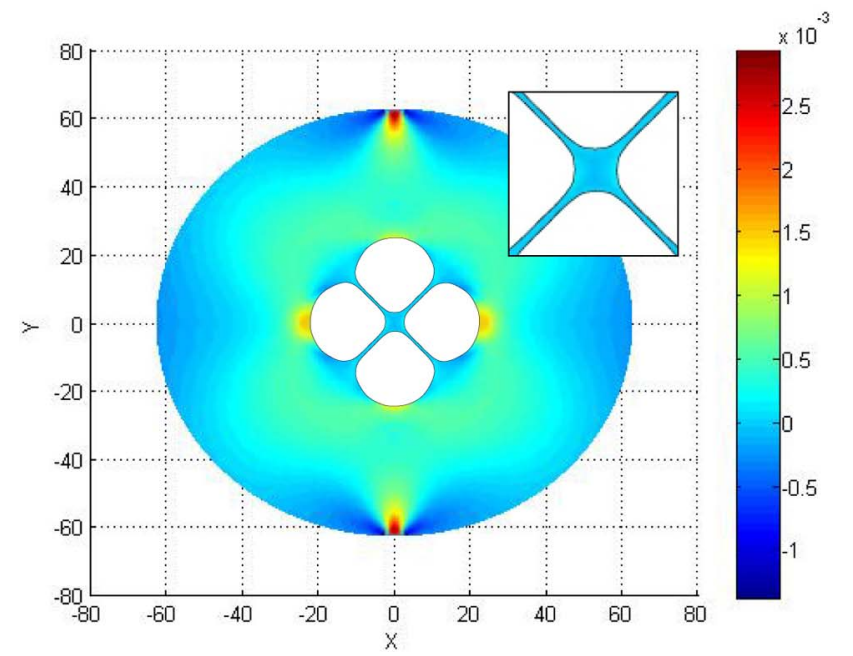

(b)

Fig. 2. (Color online) (a) Simulation of birefringence for (a) silica bridges and (b) air holes under a load of $50 \mathrm{~N} / \mathrm{cm}$. The birefringence in the fiber core region is shown as an inset. a load cell under the anvil. A broadband amplified stimulated emission source, an optical spectrum analyzer, and a circulator were used to monitor the FBG under test. The birefringence was monitored by inserting a U-bench in front of an optical spectral analyzer with a half-wave plate and a Glan-Thompson polarizer to select one of two orthogonal polarized modes. The experiment was performed under two fiber orientations to properly monitor the directional dependency on the induced birefringence. The fiber was then rotated between the two orientations under a constant load to observe the angular dependency.

The reflection spectrum of the FBG for the bridge orientation under the applied load is presented in Fig. 3(a). Under the applied transverse load, the FBG resonance peak splits into two separate from the original unperturbed state. Each peak represents one of two polarization orientations, which is confirmed by the use of a half-wave plate to select individual modes. Figure 3(b) presents FBG spectra when the air hole is oriented under the applied load. A significant decrease in the induced birefringence was observed.

The overall response of the fiber sensor to an applied transverse load is presented in Fig. 4(a) with the solid curves indicating the predicted values of induced birefringence. The experimental values match the numerical simulation when the load is less than $40 \mathrm{~N} / \mathrm{cm}$. Under the $40 \mathrm{~N} / \mathrm{cm}$, the fiber yields a sensitivity of
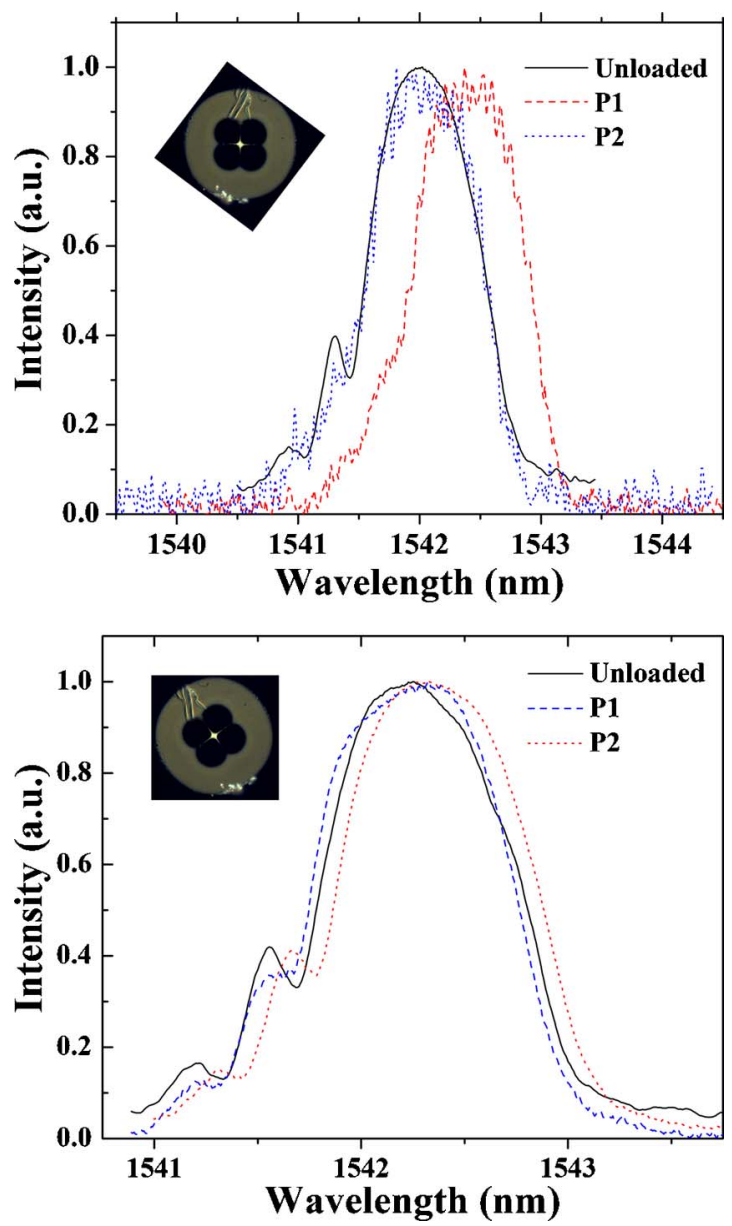

Fig. 3. (Color online) FBG peaks from two orthogonal polarizations under an applied transverse load of $47 \mathrm{~N} / \mathrm{cm}$ for the bridge under load and the air-hole under load. 

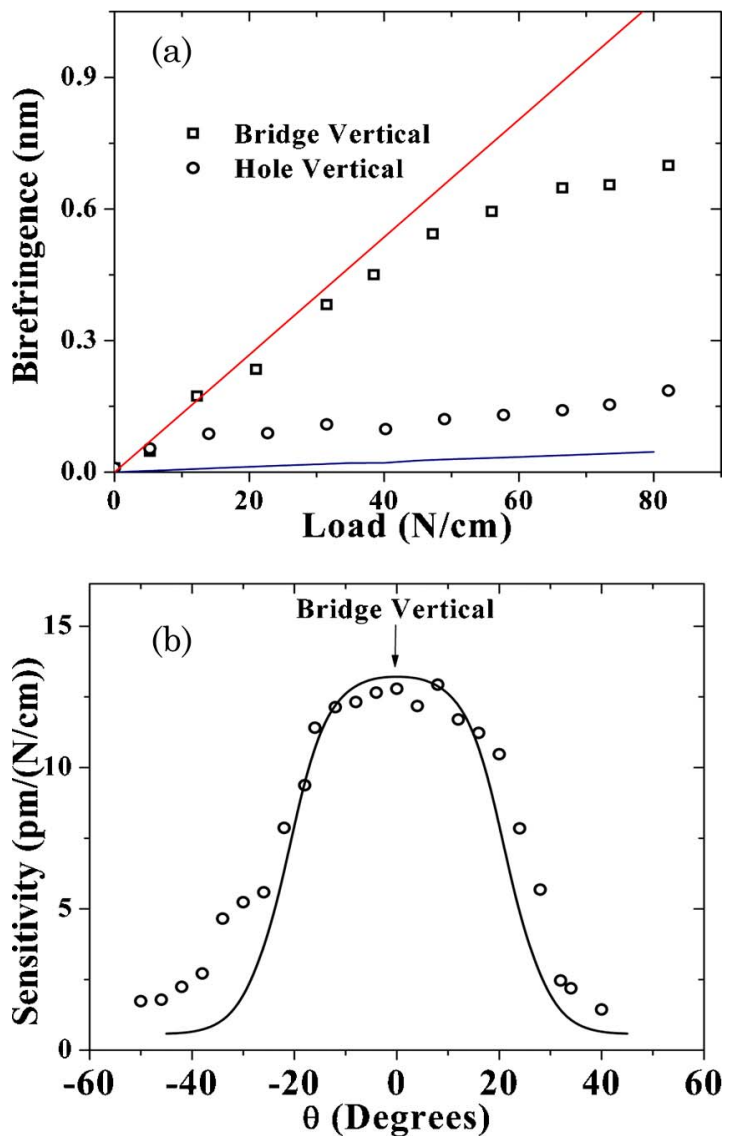

Fig. 4. (Color online) (a) FBG peak splits as functions of applied transverse loads and (b) compression sensitivity as a function of fiber orientations. The fiber is under an applied load of $47 \mathrm{~N} / \mathrm{cm}$.

$12.228 \mathrm{pm} /(\mathrm{N} / \mathrm{cm})$ when the bridge is under the load. This is in contrast to $2.19 \mathrm{pm} /(\mathrm{N} / \mathrm{cm})$ when the air hole is under the load. At higher loads, the experimental results deviate significantly from the simulation. The possible cause for this discrepancy could be that the bridge experiences a buckling at higher loads. From elasticity, the maximum load a column can withstand before buckling is the critical load. At loads higher than the critical load, the column will begin to buckle. The critical load is defined as $F_{c}=\pi^{2} E I /(K L)^{2}$, where $E$ is the Young's modulus of the material, $I$ is the second moment of inertia of the column's cross section, $L$ is the length of the column, and $K$ is the column's effective length factor and have values of $74.52 \mathrm{GPa}, 607.5 \mu \mathrm{m}^{4}, 20.33 \mu \mathrm{m}$, and 0.5 , respectively. From our geometry the critical load is $6.54 \mathrm{~N}$ (485 MPa) [12].

The angular dependency on the induced birefringence was also studied and is shown in Fig. 4(b). Because of the unique symmetry in the fiber, one orientation (bridge vertical) is more sensitive than the other (hole vertical). Experimental results show that the suspended-core fiber has a minimum sensitivity of $\sim 2 \mathrm{pm} /(\mathrm{N} / \mathrm{cm})$ when the air-holes are under load and $\sim 12 \mathrm{pm} /(\mathrm{N} / \mathrm{cm})$ when the bridge is under load. Despite this 5.58 times discrimina- tion, the contrast is significantly smaller than that of simulation results (22 times), probably due to the buckling effect. The four-hole fiber was placed under a constant load at different angles to determine the angular dependency on the induced birefringence. The simulation results predict an overall sensitivity of $0.588 \mathrm{pm} /(\mathrm{N} / \mathrm{cm})$ and $13.372 \mathrm{pm} /(\mathrm{N} / \mathrm{cm})$ at the air hole $\left(-45^{\circ}\right.$ and $\left.45^{\circ}\right)$ and bridge $\left(0^{\circ}\right)$ under the applied load. The cause of the discrepancy is currently unknown.

In summary, we have demonstrated in this Letter a directional dependent transverse load sensor using a fourhole suspended-core fiber. Resonant peak splitting of FBGs were used to measure the applied transverse load and the orientation. The fiber experiences sensitivities of 12.23 and $2.19 \mathrm{pm} /(\mathrm{N} / \mathrm{cm})$ when the bridge and hole are under the applied load, respectively. At the same time, we still can use the FBG peak shift to measure the axial strain exerted to the FBG sensors. By using the four-hole suspended-core fiber, this paper presents a FBG sensor that is sensitive to both the orientation and magnitude of transverse loads.

This work was supported by the National Science Foundation (NSF) CAREER Program (NSF0644681). C. M. Jewart is supported by the NSF IGERT Program (NSF0504345). Funding by the German state of Thuringia (Ministry of Education and Cultural Affairs) is gratefully acknowledged.

\section{References}

1. T. Geernaert, G. Luyckx, E. Voet, T. Nasilowski, K. Chah, M. Becker, H. Bartelt, W. Urbanczyk, J. Wojcik, W. De Waele, J. Degrieck, H. Terryn, F. Berghmans, and H. Thienpont, IEEE Photon. Technol. Lett. 21, 6 (2009).

2. Y. Wang, H. Bartlet, W. Ecke, K. Schroeder, R. Willsch, J. Kobelke, M. Rothhardt, I. Latka, and S. Brueckner, IEEE Photon. Technol. Lett. 21, 1450 (2009).

3. C. Jewart, K. Chen, B. McMillen, M. Bails, S. Levitan, I. Avdeev, and J. Canning, Opt. Lett. 31, 2260 (2006).

4. H. N. Xie, Ph. Dabkiewicz, R. Ulrich, and K. Okamoto, Opt. Lett. 11, 333 (1986).

5. M. Silva-Lopez, C. Li, W. MacPherson, A. Moore, J. Barton, J. Jones, D. Zhao, L. Zhang, and I. Bennion, Opt. Lett. 29, 2225 (2004).

6. C. Martelli, J. Canning, N. Groothoff, and K. Lyytikainen, Opt. Lett. 30, 1785 (2005).

7. K. Schuster, J. Kobelke, A. Schwuchow, M. Leich, M. Becker, M. Rothhardt, U. Röpke, J. Kirchhof, H. Bartelt, T. Geernaert, Proc. SPIE 6588, 658804 (2007).

8. K. M. Kiang, K. Frampton, T. M. Monro, R. Moore, J. Tucknott, D. W. Hewak, D. J. Richardson, and H. N. Rutt, IEEE Electron Device Lett. 38, 546 (2002).

9. O. Frazao, S. H. Aref, J. M. Baptista, J. L. Santos, H. Latifi, F. Farahi, J. Kobelke, and K. Schuster, IEEE Photon. Technol. Lett. 21, 1229 (2009).

10. R. Gafsi and M. A. El-Sherif, Opt. Fiber Technol. 6, 299 (2000).

11. M. Becker, J. Bergmann, S. Brückner, M. Franke, E. Lindner, M. W. Rothhardt, and H. Bartelt, Opt. Express 16, 19169 (2008).

12. S. Krenk, Mechanics and Analysis of Beams, Columns and Cables 2nd ed. (Springer, 2001), Chap. 3. 